\title{
Correction to: SEOM clinical guidelines for the treatment of head and neck cancer (2020)
}

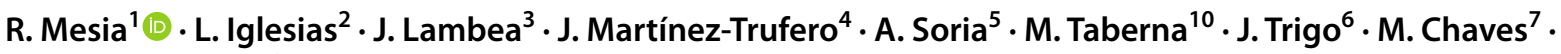 \\ A. García-Castaño ${ }^{8} \cdot$ J. J. Cruz-Hernández ${ }^{9}$
}

Published online: 11 March 2021

(c) Federación de Sociedades Españolas de Oncología (FESEO) 2021

\section{Correction to: Clinical and Translational Oncology https://doi.org/10.1007/s12094-020-02533-1}

One of the author names was published incorrectly in the original publication. The correct name should read as J. J. Cruz-Hernández.

Publisher's Note Springer Nature remains neutral with regard to jurisdictional claims in published maps and institutional affiliations.

The original article can be found online at https://doi.org/10.1007/ s12094-020-02533-1.

\author{
R. Mesia \\ rmesia@iconcologia.net \\ L. Iglesias \\ laracarmen.iglesias@salud.madrid.org \\ J. Lambea \\ juliolambea@yahoo.es \\ J. Martínez-Trufero \\ jmtrufero@seom.org \\ A. Soria \\ ainarasoria@hotmail.com \\ M. Taberna \\ mtaberna@iconcologia.net \\ J. Trigo \\ jmtrigo@seom.org \\ M. Chaves \\ manuelchavesconde@gmail.com
}

\section{A. García-Castaño}

garcicastano@yahoo.es

J. J. Cruz-Hernández

jjcruz@usal.es; jjcruz@saludcastillayleon.es
Institut Català d'Oncologia, Badalona, Spain

2 Servicio de Oncología Médica, Hospital Universitario 12 de Octubre, 12 de Octubre, Madrid, Spain

3 Servicio de Oncología Médica, Hospital Clínico Universitario Lozano Blesa, Zaragoza, Spain

4 Servicio de Oncología Médica, Hospital Universitario Miguel Servet, Zaragoza, Spain

5 Servicio de Oncología Médica, Hospital Universitario Ramón y Cajal, Madrid, Spain

6 Servicio de Oncología Médica, Hospital Clínico Virgen de la Victoria, Málaga, Spain

7 Servicio de Oncología Médica, Hospital Virgen de Valme, Seville, Spain

8 Servicio de Oncología Médica, Hospital Universitario Marqués de Valdecilla, Santander, Spain

$9 \quad$ Servicio de Oncología Médica, Hospital Clínico Universitario de Salamanca, Salamanca, Spain

10 Institut Català d'Oncologia, Hospitalet de Llobregat, Spain 